\title{
Advancing flexible volatile compound sensors using liquid crystals encapsulated in polymer fibers
}

Catherine G. Reyes, Jan P. F. Lagerwall

Catherine G. Reyes, Jan P. F. Lagerwall, "Advancing flexible volatile compound sensors using liquid crystals encapsulated in polymer fibers ," Proc. SPIE 10555, Emerging Liquid Crystal Technologies XIII, 1055500 (8 February 2018); doi: 10.1117/12.2292533

SPIE. Event: SPIE OPTO, 2018, San Francisco, California, United States 


\title{
Advancing flexible volatile compound sensors using liquid crystals encapsulated in polymer fibers
}

\author{
Catherine G. Reyes ${ }^{\mathrm{a}}$ and Jan P. F. Lagerwall ${ }^{\mathrm{a}}$ \\ ${ }^{a}$ University of Luxembourg, Physics and Materials Science Research Unit, 162a avenue de la \\ Faiencerie, Luxembourg
}

\begin{abstract}
Until recently, organic vapor sensors using liquid crystals (LCs) have employed rigid glass substrates for confining the LC, and bulky equipment for vapor detection. Previously, we demonstrated that coaxially electrospinning nematic LC within the core of polymer fibers provides an alternative and improved form factor for confinement. This enables ppm level sensitivity to harmful industrial organics, such as toluene, while giving the flexibility of textile-like sheets (imparted by polymer encapsulation). Moreover, toluene vapor responses of the LC-core fiber mats were visible macroscopically with the naked eye depending on the morphology of the fibers produced, and whether they were oriented in specific geometries (aligned, or random). We identified two types of responses: one corresponds to the LC transition from nematic to isotropic, and the other we suggest is due to an anchoring change at the LC-polymer interface that influences the alignment. While we need to study the presence that defects can have in more detail, we noted that fiber mat thickness is crucial in attempting to understand how and why we are able to visualize two responses in aligned LC-fiber mats. Ultimately, we noted that the response of the polymer sheath itself (softening) to organic vapor exposure affects the liquid crystal confinement in the core. From the microscopic point of view, this will influence the threshold concentration that fibers in a mat will overall respond to. In this paper we will discuss three findings the morphologies enabling LC-core fiber mat response to vapor seen both micro- and macroscopically, how thickness of the fiber mat can play a role in the visualization of the responses, and the effect that the polymer structure has in the mat's sensitivity threshold.
\end{abstract}

Keywords: electrospinning, liquid crystals, vapor sensing, fibers, flexible, volatile compound, non-electronic

\section{INTRODUCTION}

Ongoing research in advancing liquid crystal (LC) technologies has now exceeded well past the goal of simply developing the next electronic commercial displays. Previous review and perspective articles found in Refs. 1 and 2 , introduced us to the idea that future LC research could enhance our understanding of interactions between liquid crystal and other soft matter structures; potentially even leading us to the development of flexible, nonelectronic fiber textiles useful for the detection of harmful volatile gases. While the idea of using LC phase forming molecules as alternatives for developing layperson, user-friendly gas sensors is not so new ${ }^{3-7}$-ones which would also have form-factor versatility without the need for bulky equipment-the challenges for actually developing such materials that live up to these expectations, while simultaneously being able to endure environmental factors, have not been so trivial to overcome. Although the successful development of polymer dispersed liquid crystal (PDLC) surfaces have enriched the knowledge in determining how composites of polymers and liquid crystals can be successfully combined, still too little for too long has been known about the more chemical and thermodynamical interactions of LCs in more complex environments. It's only recently that researchers studying liquid crystals, from the applied physics and chemistry point of views, have started to uncover and exploit the non-trivial physicochemical interactions that LCs can have in various soft matter environments for enhancing organic vapor sensing.

Most notably, the works described in Refs. 8, and 9 have discussed the fabrication of specific active sensing sites which exploit the complex ionic bonding properties of both the liquid crystal phase constituents and the

Further author information: (Send correspondence to C.G.R.)

C.G.R: E-mail: catherine.reyes@uni.lu, Telephone: +352621 380430

J.P.F.L: E-mail: jan.lagerwall@lcsoftmatter.com, Telephone: +3524666446219

Emerging Liquid Crystal Technologies XIII, edited by Liang-Chy Chien, Dirk J. Broer, Igor Muševič, Vladimir G. Chigrinov, Proc. of SPIE Vol. 10555, 1055500 - @ 2018 SPIE · CCC code: 0277-786X/18/\$18 · doi: 10.1117/12.2292533

Proc. of SPIE Vol. 10555 1055500-1 
analytes of interest. In Ref. 8, the authors discuss using films of nematic LC decorated with aluminum perchlorate salt which a sarin-simulant, dimethyl methylphosphonate (dmmp), can bind to in vapor form. They found that as little as $150250 \mathrm{ppm}$ of dmmp vapor was needed to trigger an optical response in the films. In principal, the films are activated through an anchoring transition. When doped with the perchlorate salt the planar nematic film aligns to a have a homeotropic state, and when exposed to dmmp vapor, the response is characterized by a reversal of this homeotropic anchoring back to a fully planar and birefringent state. The dmmp vapor preferentially complexes to the aluminum ions and removes them from the bulk of the LC phase. Further investigations of the specific binding energies surrounding the cation-LC-dmmp relationship were carried out in 2016 for further explaining the sensing responses previously observed. ${ }^{10}$ In a similar manner, but using a specific chiral dopant rather than salt this time, the authors of Ref. 9 showed how specifically doping another nematic LC with an ester dopant responsive to water could enable the development of a color changing humidity sensor. In this case, the color change corresponded to a conformational change occurring in helical pitch of the LC as the concentration of chiral dopant in the nematic hydrolyzed in the presence of the water vapor in the surrounding environment.

While these achievements have undoubtedly served their function as proof-of-concepts for the LC field to show that viable sensing devices can be developed from liquid crystals combined with other molecules and surfaces, it is still important to consider that they demonstrate only a few dimensions of what can be addressed with liquid crystal sensing. What about the encapsulation of the LC? The combination of LCs with non-flat surfaces, or with porous materials? There are many avenues which can be explored in further engineering the LC vapor sensing concept. Some pitfalls for instance that the humidity sensor from above had were that it only functioned the fastest (within 10 minutes) when exposed to over $80 \%$ humidity, and it did not employ full liquid crystal encapsulation to ensure that the components would maintain their structural integrity during use. Likewise, the devices fabricated in Ref. 8 faced the limitation of only being characterizable via polarized light microscopy, due to the fact that the scale of the LC films were on the order of a few hundred microns. Furthermore, the addition of too much salt at the beginning of the experiment could permanently render the LC films unresponsive to the dmmp vapors, as the salt continues to solublize within the bulk of the LC as time passes.

To this end, we approach the development of responsive liquid crystal based gas sensors from a different angle - addressing the issue of flexibility and encapsulation to the LC during vapor exposure and device formation. Determining the influence of polymer encapsulation and geometry on the confinement of LCs may elucidate further understanding of how organic compound vapors can interact with its optical properties. Furthermore, we wish to understand if we can address the issue of creating a fully autonomous and flexible organic vapor sensitive fiber mat which is environmentally stable. The method we've chosen to encapsulate the LC for gas sensing is via the coaxial electrospinning technique, which results in the self-assembly of micron thick polymer fibers containing a nematic liquid crystalline birefringent core. The electrospinning process is characterized by a flowing polymer solution of significant molecular weight (usually above $10,000 \mathrm{~g} / \mathrm{mol}$ ) with a potential difference applied to it. As the solution flows toward a grounded collector the electrostatic forces on the jet lead to solvent evaporation and the solidification of polymer fibers. In this case, we have configured our spinning setup to co-flow nematic liquid crystal along with the polymer solution so that as the solvent evaporates, the LC becomes encapsulated within the final fibers. A brief schematic of the process is shown in the upper portion of Figure 1(a). A more detailed discussion regarding the optimal solution chemistries and processing parameters for co-electrospinning liquid crystals with polymers can be found in the second half of the review in Ref. ${ }^{11}$

Though the process is not complex, it enables us to fabricate a wide array of liquid crystalline core fibers with varying orientational (aligned, or random) and morphological geometries quickly (around 10-15 minutes after parameter optimization). Example polarized light microscope images of both types of morphological fiber geometries (beaded, and cylindrical) are shown in the lower half of Fig. 1(a) as a reference. In this study, we discuss the high sensitivity of our current LC core (5cb), polymer sheath (polyvinylpyrrolidone) (PVP) fiber mats to toluene vapor as a representative organic volatile compound. We chose toluene vapor because it is widely known as an industrial workplace solvent hazard which is often a byproduct for many industries formulating paints, coatings, and adhesives. According to the United States National Institute for Occupational Safety and Health (NIOSH), the recommended workplace time-weighted average (TWA), upper exposure limit for toluene 
is $100 \mathrm{ppm}$ for a 10 hour period. ${ }^{12} *$

When exposed to toluene vapor concentrations varying from $5 \mathrm{ppm}$ to $90 \mathrm{ppm}$ our LC fiber mats showed a nematic clearing response starting from about 15 to $20 \mathrm{ppm}$. In the further discussion described in section 2.3 we show how the morphological structure of the fibers can impact this exposure threshold. In section 2.2 we discuss how the fibers can also exhibit a macroscopic level response when exposed to concentrated toluene vapors (from about $3 \%$ in air). This response is characterized by a decrease in overall fiber mat scattering which is visible using polarizers, and with the unaided eye without polarizers if the fiber diameters are sufficiently large enough. In both instances of macroscopic and microscopic vapor exposure analysis the fiber mat response to toluene vapor was reversible, with the nematic LC phase returning when the toluene vapor was removed. Finally, we briefly discuss the implications that polymer structure and stability during gas sensing may have on the internal LC filling and defect formation within the fiber cores.

\section{METHODOLOGY}

\subsection{Electrospinning parameters for fabricating the LC-fiber mats}

A detailed description of all electrospinning components and solutions used for the macroscopic toluene vapor sensing experiments, along with the fabrication details of the spinneret dimensions, can be found in the experimental sections and supplementary information of Ref. 13. Additionally, a simplified schematic of the electrospinning process is shown in Fig. 1(a). For the fabrication of the LC-fibers tested for vapor sensing here, a $12.5 \mathrm{wt} \%$ polymer solution of polyvinylpyrrolidone (Sigma-Aldrich, average $\mathrm{M}_{w}$ 1,300,000)in ethanol (Carl Roth Chemicals, 96\%) was used as the sheath solution. The room temperature thermotropic nematic liquid crystal, 4-cyano-4'-pentylbiphenyl (5cb), for the fiber core was purchased from Yantai Xianhua Chem-Tech, and was confirmed to have a clearing temperature of $35.6{ }^{\circ} \mathrm{C}$ using a Linkam T95 series LTS120E temperature control stage. The concentration of ethanol and the type of $5 \mathrm{cb}$ used here were not found to cause significant changes in the quality of LC-fibers produced. Morphologically, these fibers were of the same quality of those produced in Ref. 13 at standard conditions. All electrospinning experiments for these fibers were conducted at an average temperature of $25{ }^{\circ} \mathrm{C}$ and relative humidity of $25-28 \%$, unless otherwise specifically noted. A voltage of $8-9 \mathrm{KV}$ (labeled V in Fig. 1(a)) was applied to the co-axial spinneret containing two nozzles for co-flowing the LC and polymer solution at approximately $0.5 \mathrm{~mL} / \mathrm{hr}$ and $0.9 \mathrm{~mL} / \mathrm{hr}$, respectively. The spinneret distance from the grounded, negatively charged collector was $12 \mathrm{~cm}$. Glass slides $(26 \mathrm{~mm} \times 75 \mathrm{~mm})$ were used as substrates to collect the fibers for observation and toluene sensing in the vapor cell-microscope setup, depicted in Fig. 1(b), and for the macroscopic sensing setup, shown in Fig. 1(c). The toluene used for all gas sensing experiments was purchased from Sigma Aldrich (Sure/Seal ${ }^{T M}$ anhydrous bottle).

\subsection{Macroscopic analysis setup for LC-fiber vapor exposure experiments}

To determine whether the electrospun LC-fiber mats would be visibly responsive to toluene vapors without the aid of a microscope or other electronic equipment, we exposed our samples to vapors under macroscopic polarizers approximately $20 \mathrm{~cm} \times 20 \mathrm{~cm}$ in size. A schematic of this setup is shown in Fig. 1(c). Sheets of polarizing film (Alight Polarizers) were cut and mounted at $90^{\circ}$ to one another in an elevated frame with a spacing of about $5-6 \mathrm{~cm}$. Glass slides containing the fiber mats, as shown in a representative photo close-up in Fig. 2, were placed in between the upper and lower polarizing films. The entire polarizer frame containing the fiber sample in between, was placed over a fluorescent light emitting box for illumination. A septum covered vial containing $1 \mathrm{~mL}$ of toluene was connected to a tube attached to a microfluidics unit (Fluigent, MFCS-EZ) which supplied 200 mbar of air into the headspace of the vial. The vaporized toluene was transported via another tube, also in the headspace portion of the vial, to the LC-fiber samples for sensing in between the polarizers. We estimated the vaporized toluene vapor concentration to be no more than about $3 \%$. Our estimations are shown in the supplementary information of Ref. 13.

\footnotetext{
*For California state, the TWA is actually $10 \mathrm{ppm}$, but the short-term exposure limit (STEL) is $150 \mathrm{ppm}$.
} 


\subsection{Microscopic analysis setup for LC-fiber vapor exposure experiments}

For determining the toluene vapor exposure threshold of the LC-fiber mats, a vapor exposure cell was manufactured in-house to contain the size of a typical glass slide covered with fibers. A schematic of this setup is shown in Fig. 1(b). The vapor cell was made to hold a volume of about $15 \mathrm{~mL}$, including the respective tubings at the inlet and outlet. Organic vapors can be introduced into the cell using one of two methods - either the inlet is connected to a 1L syringe (shown on the right side of the vapor cell in Fig. 1(b)), or an inert gas sampling bag containing the vapors via the push/pull lock located on the bag itself. Irrespective of the method used for vapor introduction, toluene vapors of specific ppm level concentration were formulated the same way using the $1 \mathrm{~L}$ syringe. Using the appropriate volume range of Eppendorf pipettes (Sigma Aldrich) various $\mu \mathrm{L}$ volume amounts of toluene were separately inserted into the gas tight 1L syringe (Hamilton Company, Model S1000 TLL) at room temperature and at about $32 \% \mathrm{RH}$. The liquid toluene was left to evaporate until the vapors completely dispersed throughout the sealed syringe barrel. Tab. 1 shows the corresponding concentrations of vapor made from the toluene dispersed.

Afterwards, the toluene vapors were either transferred directly into the vapor cell, or transferred via inert PTFE tubing into 1L gas sampling bags (Tedlar 1L-Push/Pull Lock Valve, Sigma Aldrich). An advantage of using the Tedlar bags is that the vapor generated can be stored in a secure manner for weeks at a time without risk of contamination or leaking. The vapor cell attached to either the filled syringe, or the Tedlar bag, was then placed over the stage of the microscope, and a region of interest was chosen for observing the relative changes in LC brightness intensity as the sample was exposed to vapors. A green filter ( $\lambda: 525 \mathrm{~nm}$, Zeiss) was placed over the bottom polarizer of the microscope so that the intensity changes could be tracked by a single wavelength of light. Recordings of the fiber responses to the toluene vapor concentrations in the cell were done using a Canon EOS 706 camera. Time-lapse images of the vapor-fiber responses were extracted from the videos. These green light filtered RGB images of the fibers were then batch converted to 8-bit grayscale by isolating the green channel portion using a "split channels" macro programmed in ImageJ 1.51s (NIH, USA http://imagej.nih.gov/ij). The images were thresholded to find the mean gray value changes in brightness versus time, and then normalized to the mean gray values before exposure at highest brightness. The average area of observation possible using a 10x objective with the vapor cell was $\sim 488 \mu \mathrm{m}$ x $868 \mu \mathrm{m}$. A Nikon LV100 polarized optical microscope with 10x objective was used for all microscopic vapor analysis experiments.

Table 1. Toluene volumes for creating the corresponding vapor concentrations in ppm and mol\% in $1 \mathrm{~L}$ of air for the microscopic sensing experiments. The mol\% concentrations are calculated assuming relatively dry air (below $40 \% \mathrm{RH}$ ).

\begin{tabular}{|l|l|l|}
\hline Volume $(\mu \mathrm{L})$ & Vapor Concentration $(\mathrm{ppm})$ & Concentration $(\mathrm{mol} \%)$ \\
\hline 5 & 5 & 0.11 \\
\hline 10 & 10 & 0.23 \\
\hline 15 & 15 & 0.34 \\
\hline 20 & 20 & 0.46 \\
\hline 50 & 50 & 1.13 \\
\hline 90 & 90 & 2.02 \\
\hline
\end{tabular}

\section{RESULTS AND DISCUSSION}

\subsection{Beaded fiber mats respond macroscopically without the need for polarizers to aid visualization}

In our previous study, ${ }^{13}$ we had examined various coaxial LC-polymer fiber type configurations and morphologies that allowed the facile visualization of the toluene vapor sensing response at the macroscale level. We had found that electrospinning at either high relative humidity (over 60\%), or with high LC flow rate (15:9 ratio LC:polymersolution), resulted in coaxial fibers with highly beaded morphologies-such as those shown in the bottom half of the green inset in Fig. 1(a). We had also observed that an accumulation of these beaded fibers over one 
another, in turn, caused the formation of highly meshed, and flattened fiber mats. Alternatively, electrospinning at conditions with low relative humidity (around 25\%), and with a lower LC flow rate (5:9 ratio LC:polymersolutions), resulted in the formation of more cylindrical type fibers without beading. In that study we attributed this difference in morphological structures to the excess in lower molecular weight fluid buildup in the Taylor cone and fiber forming jet. Under non-humid conditions, or conditions when the polymer solution flow rate is higher than the core, the PVP polymer is the only component solidifying during the coaxial electrospinning process, presumably losing ethanol to evaporation as time passes and the whipping jet becomes thinner and thinner until finally reaching the collector as a solid micron sized continuous fiber. During the solidification process and charges that are built on the surface of the polymer jet from the potential applied, the Rayleigh instability of the non-evaporating components within is suppressed; the $5 \mathrm{cb}$ LC in this case, if flowed at a corresponding rate will conform to fill the narrow confinement within the solidifying fibers without distorting the polymer sheath. However, even if the time for spinning is kept the same, this process dramatically changes when a higher flow of either $5 \mathrm{cb}$, or excess water vapor (for the case of high humidity) is introduced. Rather than a regular evaporation of ethanol from the polymer sheath occurring, and the core component remaining largely negligible to the process, there will now be a more pronounced fluctuation of ethanol and water entering the polymer sheath, slowing down the sheaths' solidification, and potentially affecting how the core component fills this new sheath structure forming.

One of the most pronounced consequences will be that the new sheath structure will take on a beaded form as the excess in fluid in the core, and the destabilized evaporation rate of the polymer solvent, enable the favorable Rayleigh instability regime to take over the process. The meshed fiber mats that formed in our previous study were a direct consequence of poorly solidified (and still very wet) polymer sheaths which have stretched to a thinner state in order encapsulate a far larger quantity of liquid per segment. On average, the beaded fibers can have beaded sections as wide as $20 \mu \mathrm{m}$ in diameter, as opposed to the cylindrical fibers which are usually closer to $2-3 \mu \mathrm{m}$ in thickness. Furthermore, in the supplemental information of Ref. ${ }^{13}$ we estimated this difference in sheath thickness to be about an order of magnitude; the cylindrical fibers having a sheath that is about $30 \%$ thicker overall than the sheath of the beaded fibers.

Arguably, while the applied voltage to the system in this case may be raised to overcome any instabilities in the jet, there is no guarantee that the increase in potential will not simply cause the jet to solidify prematurely mid-flight on its way to the collector, causing a rubbery film, rather than fibers to develop instead. We are currently investigating and publish on some of these more intriguing effects that result from the mixing of polymer solution, solvent, and liquid crystal when subjected to highly humid environments and high potentials.

\subsection{Cylindrical aligned fiber mats show two responses only visible with polarizers}

Most importantly, although the scale of both types of LC filled fibers (beaded and cylindrical) are still well below $80 \mu \mathrm{m}$, the average thickness of a human hair for comparison, these morphologies have a crucial impact on how the toluene vapor sensing responses develop within the final macro-scale fiber mats formed. Interestingly, it was the beaded fiber mats which were able to show responses to vapor exposure without the need for macroscopic polarizers and light box. A decrease in fiber mat scattering was observed at the site of toluene vapor exposure consistent with the clearing of the LC nematic phase in the fiber cores. This is shown in Fig. 3(a-f), where the original scattering of a fiber mat produced with high LC flow rate (a-c), and the original scattering of a fiber mat produced at high humidity (d-f), reduce when placed over a colored background. Microscopically, under crossed polarizers, we observed an almost immediate loss of birefringence in the LC-fibers when the vapor exposure tube was brought near to the fiber mat. Upon removal of the toluene vapor, the birefringence, and original macroscopic fiber mat scattering, gradually returned. For all other cylindrical fiber mats electrospun in both aligned and random mat configurations, the macroscopic changes in scattering were not visible without the aid of polarizers. Again, we postulated that this was due to the reduced individual fiber thickness, the lower quantity of LC available per fiber segment, and the likelihood that sheath was thicker during its formation than that of the beaded fibers.

For the aligned cylindrical fiber mat configuration, however, again we found another surprising result. When exposed to toluene vapors under crossed macroscopic polarizers, the aligned, cylindrical LC-fiber mats showed 2 types of vapor responses. Fig. 4(a-b) show updated time-lapse frames of the same aligned fiber mat sample placed 
in between crossed polarizers and exposed to toluene vapors in two directions; at $0^{\circ}$ to the bottom polarizer (a), and at $45^{\circ}$ (b) for maximum LC brightness. As with the initial mention of these observations, first described in Sections 3.1 and 4.1 of Ref., ${ }^{13}$ the vapor responses seen for this fiber mat type here also varied in scattering intensity based on the orientation of the aligned fiber mat to the polarizers. At an orientation of $0^{\circ}$ to the bottom polarizer the aligned fiber mat sample shows an immediate response when exposed to the vapors. On average, from Fig. 4(a), it takes about 1.5 seconds for the response from one exposure sweep to fade; the last image in this row taken at 7.8 seconds showing the original ground state scattering of the mat in this horizontal position. Turning the sample by $45^{\circ}$, the base scattering of the sample increases dramatically as the LC in the fibers is aligned for maximum brightness, assuming that the LC is aligning along the fiber axis direction. At this orientation, there is a visible secondary response that is visible in addition to the original 1st response, which is now understandably much more muted than before when the sample was aligned at $0^{\circ}$ to the polarizers. A distinction between the area of coverage that both responses take up in Fig. 4(b) is made from the red and blue outlines. The same broadly sweeping fast response occurring in row (a) is less apparent in (b), but from the way this broad response layers over the second more contrasted and localized response shown in blue (which only appears about 2 seconds later after prolonged exposure), we notice that it disappears faster than the more contrasted response. The contrasted response in blue takes about another 8 seconds to dissipate long after the removal of the toluene vapors.

While it's only possible to view the more contrasted response of the aligned fibers when they are oriented at $45^{\circ}$, it is interesting that both responses (the broadly sweeping one, and the localized one) are actually occurring in both orientations of the aligned fiber mats. While at $45^{\circ}$ we are able to detect both responses, when the mat is reoriented to $0^{\circ}$ with respect to the bottom polarizer, we are only able to detect the broadly sweeping scattering response. This further shows that while the two responses can occur together, they affect individual fibers independently at different rates. Thus, we still hypothesize that the internal LC core alignment in the fibers may play a role in showing the two different responses. While its true that most of the LC aligns along the fiber axis for the majority of the fibers, areas of highly strained nematic (perhaps exhibiting a high saddle-splay constant due to the $23 \mu \mathrm{m}$ thick cylindrical fiber confinement) may also exist near the fiber sheath surface. This can be a plausible explanation for the broadly sweeping vapor response seen to quickly occur and vanish upon vapor exposure and removal. Meanwhile, the localized, more contrasted response could actually be the nematic bulk of the fibers clearing to the isotropic phase, which does take slightly longer to develop upon initial vapor exposure.

Of note is that in the previous experiments of Ref., ${ }^{13}$ the polarizing films used were of lower polarizing quality and the frame on which they were mounted had a large spacing $(15 \mathrm{~cm})$ which was responsible for a higher throughput of surrounding unpolarized light. Thus, the cross-polarized background for the macroscopic sensing experiments was very light blue in color rather than achromatic. At the time, this did not allow us to clearly visualize the fact that the aligned fiber mats showed both scattering responses occurring at once at the $45^{\circ}$ position. However, with the polarizer frame reconstructed to have a lower separation distance from the sample (about $6 \mathrm{~cm}$ ), and the polarizing films themselves replaced to have less light transmission when crossed, the aligned macroscopic sensing experiments here revealed a truer contrast in the scattering responses than before.

\subsection{The thickness of the LC-fiber mats impacts vapor response visibility}

Lastly, in addition to considering our hypothesis that the aligned fiber mats can have two types of vapor responses possibly due to a difference in nematic core alignment, we also hypothesize that the thickness of the fiber mats and the quantity of electrospun fiber layers deposited over each other may also play a crucial role in the visualization of these two responses. Fig. 4(c) shows an image series of a thicker aligned fiber mat (electrospun for about 15-20 seconds longer than the fibers shown in (a)) that is exposed to the same quantity of toluene vapor in the same manner as described above. When the sample is oriented at a $45^{\circ}$ angle for maximum LC brightness and exposed to vapor, the quickly occurring broader response appears to be extremely faint, and is hardly noticeable at all. In fact, the more contrasted response is the most easily observed, yet it also appears more localized in comparison to the images in rows (a) and (b). It appears as though that the fiber layers closer to the bottom of the glass substrate show the broader scattering response most clearly, and that the upper fiber layers mask the effect. In the previous study ${ }^{13}$ we had ruled out the possibility that the glass surface might be influencing the scattering responses to vapor by repeating the sensing experiment with freely hanging LC-fiber mats, and also 
non-LC filled pure polymer fiber mats the latter control experiment was performed again this time with the newer polarizers and there is still no detectable change in the scattering intensity of the fiber mat. Thus, when it comes to the thicker LC-fiber mats, it is more likely that the toluene vapors are penetrating the various fiber layers that make up the mat as a whole at different rates, and the observation of the scattering responses seen are the average result of multiple areas clearing and relaxing at once.

For instance, if the fiber mat is 4 layers thick, and the vapor is passed over the sample one time in a fast motion, while the upper 2 layers may have many fibers with LC that clears, or changes in alignment, the bottom 2 layers may not. Because the bottom two layers of fibers are not affected by the vapor exposure, and retain their original scattering the observation of effects on the first two fiber layers may not be so visible if the bottom two layers compensate in optical properties. Likewise if the toluene vapor is passed multiple times over the same area of the thick sample, while the bottom layers of fibers respond, the upper layers might be relaxing back to their original scattering state, or vice-versa. Studies comparing fiber mat thickness and response threshold to the vapors may provide more insight into how the level of visibility of the two responses in the fiber mats can change.

\subsection{Differences and similarities in the quantified responses of two types of LC-fiber mats responding to toluene vapors}

In addition to the fact that the LC-fiber mat thickness may be an important factor contributing to the level of visibility of the macroscopic scattering responses, we have also observed that the fiber mat quality itself plays an important role in the time it takes for a response to develop. If the fiber mat is subjected to a humid environment $(\geq 45 \%)$, or excessive trials of vapor exposure, then the fiber mat appears to take somewhat longer to respond after a certain threshold, about $15-20$ ppm, has been reached. Compare the fiber areas from Fig. 5 and Fig. 6 for instance. These fiber areas were both measured using the same approach shown in Fig.1(b) and discussed in Section 2.3. The fiber area observed for vapor sensing in Fig. 5 have fibers that start off with a much higher level of brightness and visible birefringence than those of Fig. 6. If one observes closely in the corresponding uncrossed polarizer images of these two mat areas, the fibers in Fig. 6 show visible signs of merging and meshing, with also some evidence of thin films of LC and polymer sheath forming in between the crossed points of several fibers. It's possible that this could just be the LC leaking from in between the two fibers having merged, but more than likely, because PVP is known to be moisture sensitive, it's probably a combination of both the liquid crystal and softened polymer spilling in between the crossed areas of merged fibers. A significant difference between the conditions which the fibers from Fig. 6 have been kept, versus those in Fig. 5, is that the former mat been exposed to a slightly more humid environment for an extended period of time after production (roughly $45 \%$ ambient $\mathrm{RH}$ ) and furthermore had been exposed repeatedly to about twice as many more trials of toluene vapor than the mat shown in Fig. 5 above. The fiber mat measured in Fig. 5, on the other hand, had only been exposed to toluene vapors a total of four or five times, and was kept in a slightly drier ambient conditions (about $35 \% \mathrm{RH}$ ). Despite the fact that this fiber mat area in Fig. 5 contains a some fibers with beaded sections; sections where we have reason to believe that the polymer sheath is significantly thinner than for the cylindrical segments, there are no areas where the fibers cross where any visible filament meshing or LC leaking can be visibly seen to have occurred. Another important aspect to note is that many of the fibers in this mat, as opposed to the one shown in Fig. 6, are on slightly different planes of focus. On average, there is roughly a $5-10 \mu \mathrm{m}$ spacing between these fibers, so it's rather unlikely that they are in much contact with each other.

As to why the fiber mat shown in Fig. 6 has much less LC birefringence than the fibers in Fig. 5, the reason likely stems from the fact that the polymer sheath had swelled with too much exposure to moisture and toluene vapor. As PVP is already hygroscopic, it's possible that the polymer chains had softened to the point where excess moisture could no longer evaporate from the sheath, and allowed the LC within to move in between the wet polymer structure more freely. This can have an effect on the rate of changing brightness intensity values after the threshold points shown on the respective plots in Fig. 5 and Fig. 6. For instance, 90 ppm of toluene vapor caused a slower and prolonged drop in LC brightness in the merged fibers at 3 minutes compared to those in the sample above in Fig. 5. Unlike the fiber area in Fig. 5, the fibers in Fig. 6 do not vary in focus, they are all on the same plane, and probably in their repeated toluene exposure became flattened and ribbon-like in cross-section, rather than truly remaining cylindrical. This flattening in turn may have contributed to the 
spreading of the polymer structure on the glass substrate, both preventing the fast clearing of the LC in the fiber cores and, with the thinning of the polymer sheath, the leaking of LC in between the fiber crossed points.

As far as the threshold concentration needed to cause a visible response in the fiber mats, it appears that both mats, even though they are from different samples, share the threshold range of about $15 \mathrm{ppm}-20 \mathrm{ppm}$. It's also possible that even the flattened mat from Fig. 6 may show a slightly lower threshold from slightly lower than $5 \mathrm{ppm}$ due to the thinner areas in the fiber mat, that have either merged, or have LC and polymer, that may have leaked or flattened out. It's important to note, however, that if this true, then it really shouldn't be interpreted to mean that meshed fiber mats are potentially better than the non-meshed or non-leaking fiber mats at vapor sensing-for while the former may have a lower vapor concentration threshold for response, they are certainly far less robust and versatile than the latter, simply because the polymer structure which encapsulates the LC has clearly been adversely and irreversibly altered by environmental conditions and repeated use. These latest findings clearly demonstrate that in order to have a more reliable estimate of the threshold response range that electrospun LC-fiber mats can have, various mat thicknesses need to be measured to see how their responses vary with time, along with the sheath to determine if there are signs of cracking or softening.

\section{CONCLUSIONS}

In this review and update regarding our work on electrospun LC-fiber mats for volatile compound (toluene) sensing, we have asserted the points that individual fiber strand morphology, and the macroscale mats that result from a compilation of these many strands, play crucial roles in how the vapor responses are observed. While beaded fiber mats contain more LC in the individual fiber cores, having caused the polymer sheaths to thin out and strain in the process of encapsulating this larger quantity of material than for the cylindrical fiber case, they have the advantage of displaying macroscopic responses to vapor exposure that are fully detectable by the naked eye without a user's need for polarizers or extra illumination. On the other hand, while the cylindrical LC-fiber mats have responses to vapor that are macroscopically detectable only through the use of polarizers, due to their more solid, thicker polymer sheaths and narrower individual fiber strands, when collected in an aligned configuration, we see that there can be two distinct levels of vapor response visible if the mat is tilted from $0^{\circ}$ to $45^{\circ}$. These two levels of vapor response can give us further clues as to how the LC in the fiber cores may be collectively and individually responding. While at first it may macroscopically appear that the responses in the LC-fiber mats are the result of the nematic to isotropoic transition occurring within the LC cores, the observation of two types of responses occurring within the aligned configuration of LC fiber mat brought us to the speculation that there can be regions in the individual fiber strands that do not have the regular planar alignment we had assumed. If this true, then perhaps a quickly occurring alignment change can be responsible for the response visualized in the $0^{\circ}$ orientation case, and then the clearing response for the second response seen in the $45^{\circ}$ case.

While it is still necessary to perform a detailed analysis of how distinct regions of the fibers, exhibiting many LC defects, behave specifically under vapor exposure, we also found that fiber mat thickness (referring to the amount of fiber layers present in our mats) play a role in how the responses are perceived. Thicker mats, created by electrospinning for a slightly longer period of time, exhibit much more muted responses than the thinner aligned mats. This may be due to the fact that the same amount of vapor cannot penetrate the entirety of the mat at the same time due to the multiple layers present. And perhaps while a quickly occurring alignment switch might be happening on the first few fiber layers, the response appears muted to the eye for two reasons; first, because the ground state scattering of the sample has increased and second, the vapor, thereby causing the alignment switch, may not be extending to the fiber layers below. Finally, our microscopic threshold analysis showed that fiber mats with considerably more flattened fibers, and fibers with noticeable leaking and meshing, had shown a lower ppm threshold concentration than the fibers which were not leaking or meshed ( $5 \mathrm{ppm}$ in the former, versus $15 \mathrm{ppm}$ in the latter). At the same time, there were significantly lower levels of starting LC brightness and birefringence in the meshed and leaking fibers. This leads us to believe that if the polymer encapsulation is playing a role in how the vapor sensing responses in the LC-fiber mats are perceived macroscopically, it is also playing a role in changing the microscopic LC response threshold, as the LC may also be re-adopting its configuration within a changing polymer structure with time. 


\section{ACKNOWLEDGMENTS}

Financial support from the University of Luxembourg and the European Research Council (ERC, Consolidator Project INTERACT, grant number 648763) is gratefully acknowledged.

\section{REFERENCES}

[1] Lagerwall, J. P. F. and Scalia, G., "A new era for liquid crystal research: Applications of liquid crystals in soft matter nano-, bio- and microtechnology," Current Applied Physics 12(6), 1387-1412 (2012).

[2] Kim, D. K., Hwang, M., and Lagerwall, J. P. F., "Liquid crystal functionalization of electrospun polymer fibers," Journal of Polymer Science Part B: Polymer Physics 51(11), 855-867 (2013).

[3] Jacobs, J. E., "Liquid Crystal Gas Analyzer," USPTO Patent: 3927977, 1-7 (1975).

[4] David, D. J. and Hardy, E. E., "Organic Vapor Detection with Liquid Crystals," USPTO Patent: 4040749 , 1-7 (1977).

[5] Dickert, F., Haunschild, A., Hofmann, P., and Mages, G., "Molecular recognition of organic solvents and ammonia: shapes and donor properties as sensor effects," Sensors and Actuators B: Chemical 6(1-3), 25-28 (1992).

[6] Nicholas, P. and Mccollum, T., "Liquid Crystal Sensors," WIPO International Patent: 9932878, 1-44 (1999).

[7] Mujahid, A., Stathopulos, H., Lieberzeit, P. A., and Dickert, F. L., "Solvent Vapour Detection with Cholesteric Liquid CrystalsOptical and Mass-Sensitive Evaluation of the Sensor Mechanism," Sensors 10(5), 4887-4897 (2010).

[8] Hunter, J. T. and Abbott, N. L., "Dynamics of the chemo-optical response of supported films of nematic liquid crystals," Sensors and Actuators, B: Chemical 183, 71-80 (2013).

[9] Saha, A., Tanaka, Y., Han, Y., Bastiaansen, C. M. W., Broer, D. J., and Sijbesma, R. P., "Irreversible visual sensing of humidity using a cholesteric liquid crystal.," Chemical Communications (Cambridge, England) 48(38), 4579-81 (2012).

[10] Roling, L. T., Scaranto, J., Herron, J. A., Yu, H., Choi, S., Abbott, N. L., and Mavrikakis, M., "Towards firstprinciples molecular design of liquid crystal-based chemoresponsive systems," Nature Communications $\mathbf{7}$, 13338 (2016).

[11] Urbanski, M., Reyes, C. G., Noh, J., Sharma, A., Geng, Y., Subba Rao Jampani, V., and Lagerwall, J. P. F., "Liquid crystals in micron-scale droplets, shells and fibers," Journal of Physics: Condensed Matter 29(13), 133003 (2017).

[12] United States Dept. of Labor, "OSHA Occupational Exposure Limits - Toluene," (https :

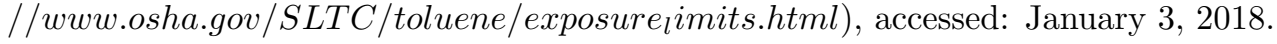

[13] Reyes, C. G., Sharma, A., and Lagerwall, J. P. F., "Non-electronic gas sensors from electrospun mats of liquid crystal core fibres for detecting volatile organic compounds at room temperature," Liquid Crystals 43(13-15), 1986-2001 (2016). 
(a)

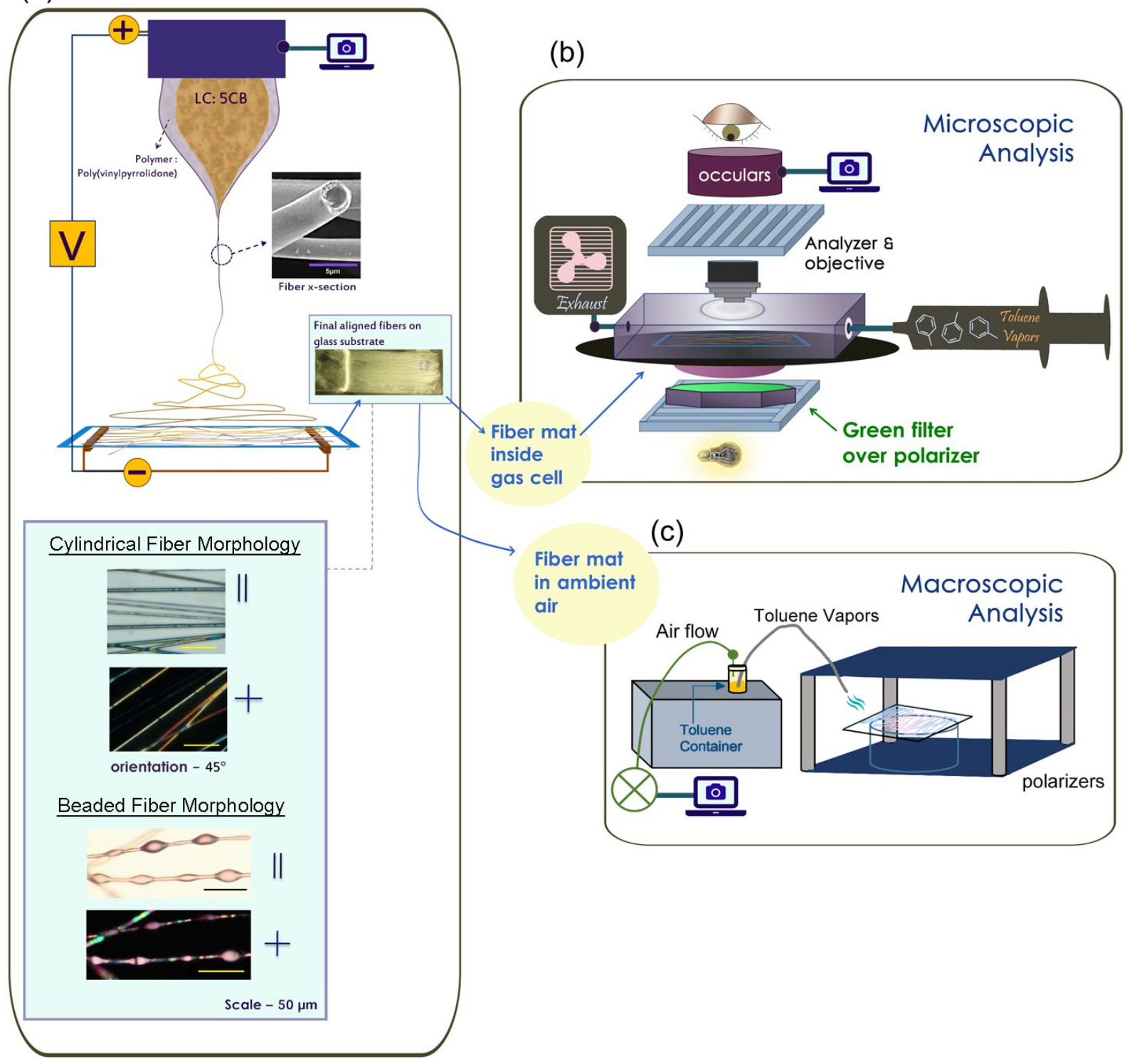

Figure 1. General schematics showing the coaxial electrospinning process for creating LC-core filled polymer fibers (a), and the vapor sensing setups used for evaluating the LC-fiber response microscopically (b), and macroscopically (c). The LC-polymer fibers electrospun onto the negatively charged collector and are directed to fall in an aligned manner from the electrodes placed on the collector substrate. An example of a glass slide containing aligned fibers is shown in the small green box in (a); the fibers can either be cylindrical or have beads and are shown, microscopically under aligned and crossed polarizers, to have birefringent LC cores (scale bars are $50 \mu \mathrm{m}$ ). These fiber mats can either be placed in a gas cell for controlled exposure to volatile organic vapors (b), or placed under large cross polarizers for macroscopic evaluation in ambient air conditions. 


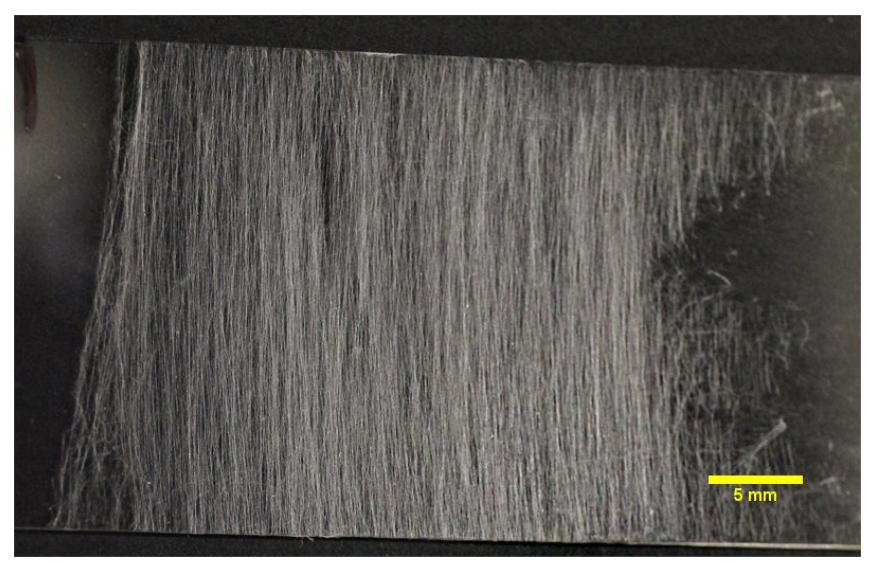

Figure 2. A representative sample of the electrospun fiber mat used for vapor sensing experiments collected on a glass slide. The sample predominantly contains aligned fibers, roughly $2-3 \mu \mathrm{m}$ in diameter, and was laid over a black background for easily viewing the fiber mat texture. The scale bar reads $5 \mathrm{~mm}$.

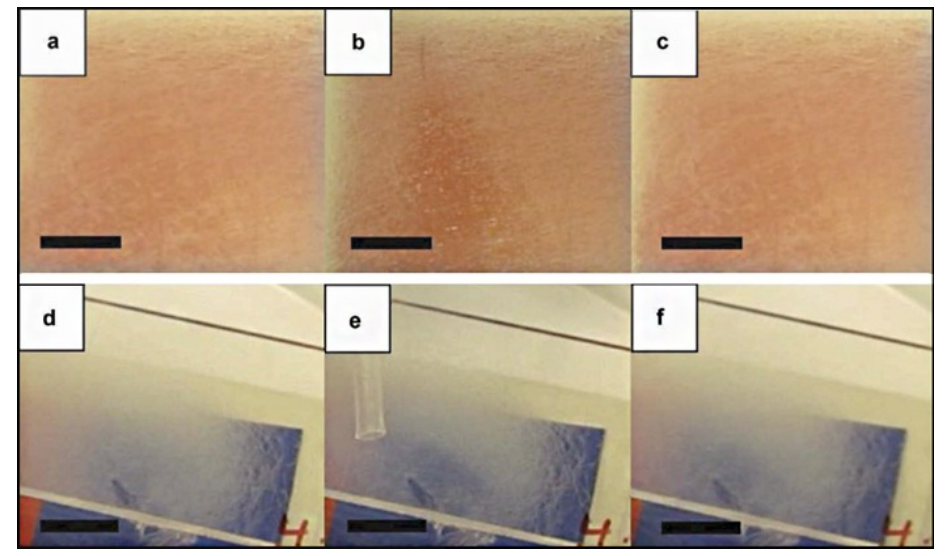

Figure 3. Macroscopic images, obtained without polarizers, of coaxial PVP-LC fiber mats spun at $25 \%$ relative humidity with a 15:9 LC:PVP flow rate ratio before (a), during (b) and after (c) gas exposure. The second row shows coaxial PVP-LC fiber mats spun at $61 \%$ relative humidity with a 5:9 LC:PVP flow rate ratio, before (d), during (e) and 13 s. (f) after gas exposure. Scale bars: $10 \mathrm{~mm}$. Figure is taken from Ref. 13; permission: CC BY-NC-ND 4.0 
(a)
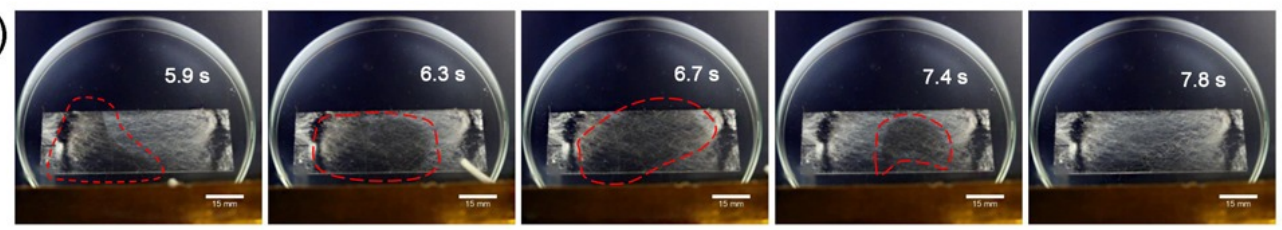

(b)
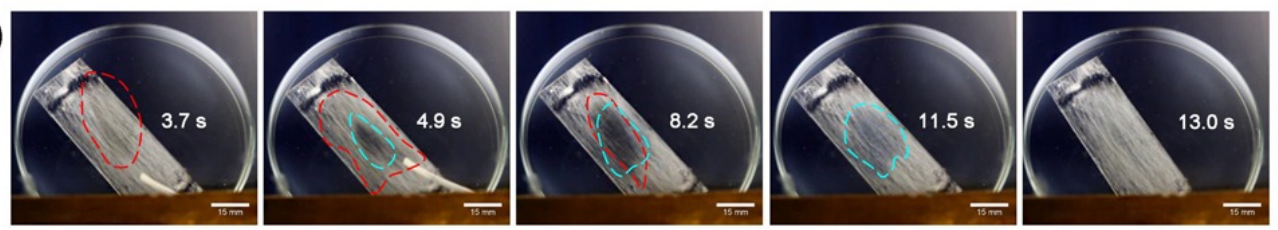

(c)
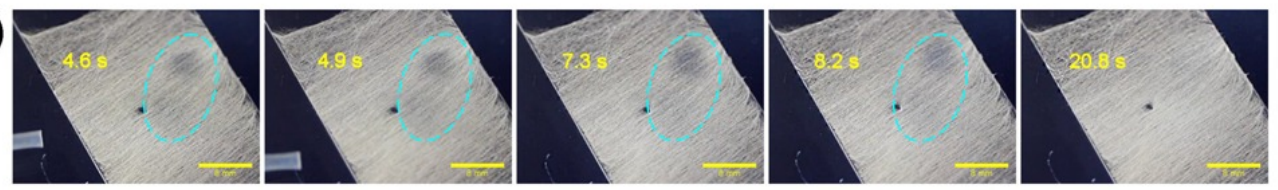

Figure 4. Updated macroscopic time-lapse experiments of aligned LC-fibers exposed to toluene vapors using the experimental setup in Fig. 1(c). Aligned fiber mats were oriented at $0^{\circ}$ (a), and $45^{\circ}$ (b, c) to the bottom polarizer. The images shown in (a) and (b) are from the same fiber mat. The images in (a) show a fast, broad scattering response occurring within about 1.7 seconds of exposure, outlined in red. The images in (b) show an additional response that starts about 2 seconds after initial exposure, outlined in blue, and lasts until 8 seconds after the vapor is removed. Row (c) shows how the level of visibility of the vapor responses decrease dramatically in a thicker fiber mat spun - the fast broad response from (a) is no longer visible. (note: there is a small tear in the center of this mat which remains constant, and is not affected by the vapor exposure). Scale bars for (a) and (b) read $15 \mathrm{~mm}$, and $8 \mathrm{~mm}$ for (c). 

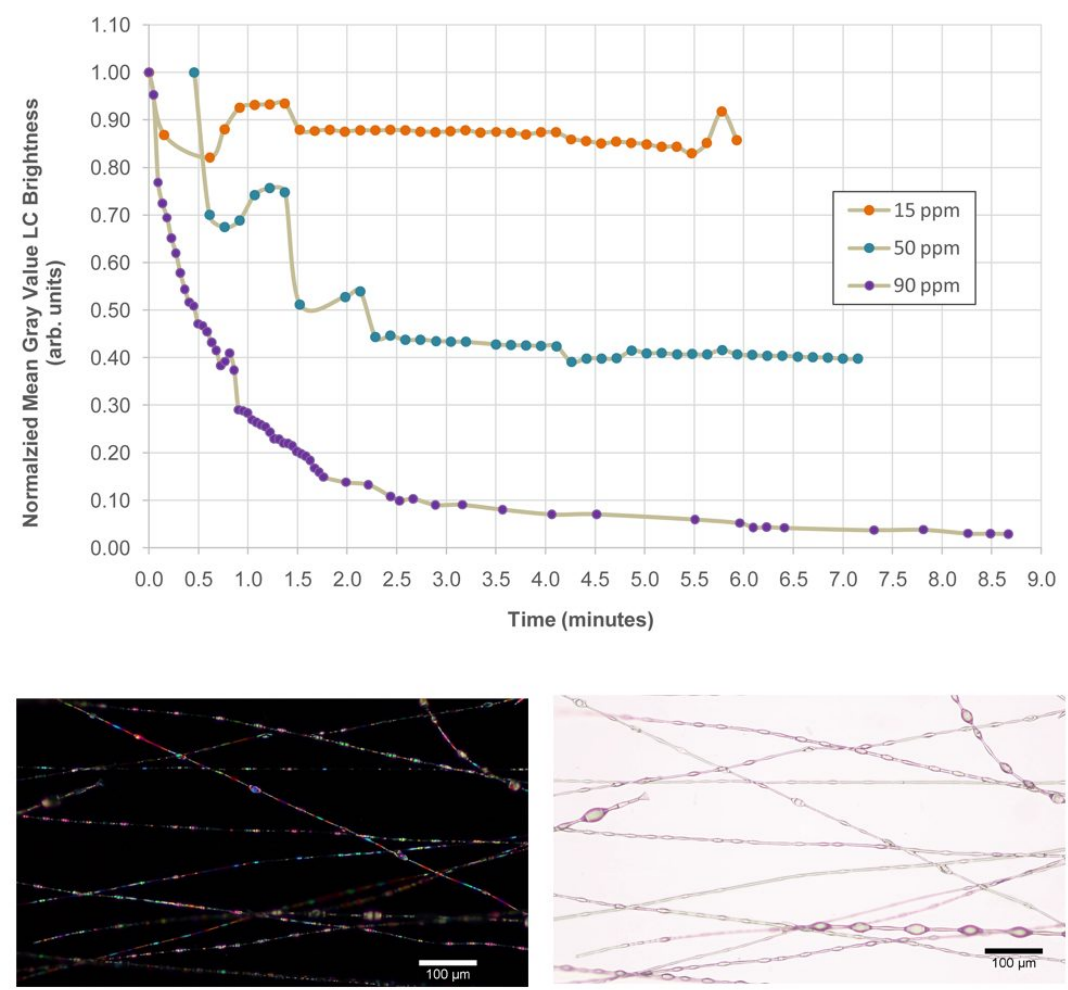

Figure 5. Quantified responses observed for fibers exposed to toluene vapor using the microscopic analysis approach described in Fig. 1(b). The normalized mean gray values obtained from recording the changing LC birefringence in the fibers were plotted against time elapsed in minutes. The concentrations tested were 15, 50 and 90 ppm. Each point is the average of over 200 regions-of-interest in the fiber mat area measured. The fibers were not held in a humid environment prior to vapor exposure. The microscope images below show the area measured with and without crossed polarizers. (scale bar: $100 \mu \mathrm{m}$ ) 

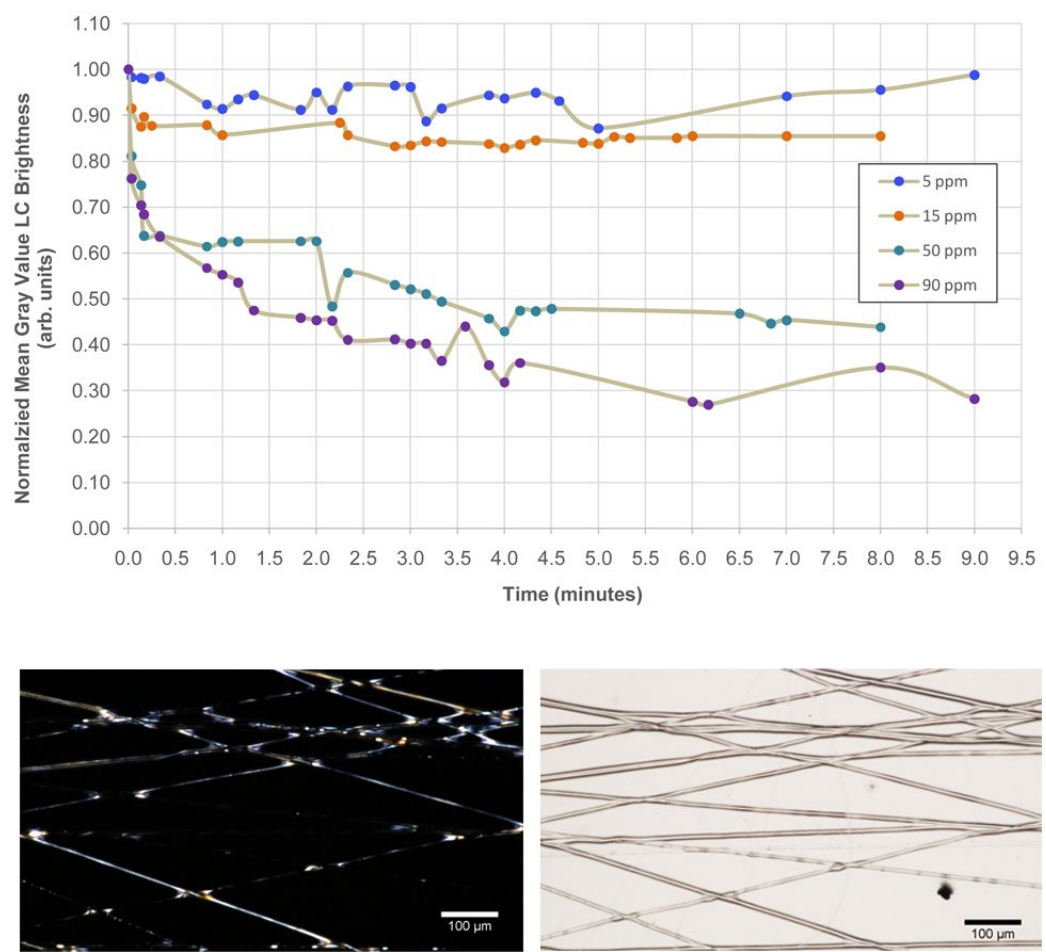

Figure 6. Quantified responses observed for fibers exposed to toluene vapor using the microscopic analysis approach described in Fig. 1(b). The normalized mean gray values obtained from recording the changing LC birefringence in the fibers were plotted against time elapsed in minutes. The concentrations tested were 5, 15, 50 and 90 ppm. Each point is the average of over 200 regions-of-interest in the fiber mat area measured. The fibers had already been held in a humid environment for some time prior to vapor exposure. The microscope images below show the area measured with and without crossed polarizers (scale bar: $100 \mu \mathrm{m}$ ) 